\title{
Priming Attitudes toward Feng Shui
}

\author{
Wendy W. N. Wan \\ Sun Yat-Sen University \\ Peiguan $\mathrm{Wu}$ \\ Sun Yat-Sen University \\ Chung-Leung Luk, \\ City University of Hong Kong \\ Kim-Shyan Fam \\ Victoria University of Wellington \\ Jessie J. X. Lou, \& H. Xu \\ City University of Hong Kong
}

\begin{abstract}
Previous research has identified three fundamentally different attitudes toward Feng Shui: the instrumental view, the spiritual view, and the minimalist view. An experiment was conducted to test the prediction that each of these three attitudes can be activated in people by "priming" them with related concepts. As predicted, priming people with concepts to do with uncertainties and ups and downs in life led them to a higher level of agreement with the instrumental view of Feng Shui. Priming people with concepts of nature and cultural identity led to a higher level of agreement with the spiritual view of Feng Shui. Finally, priming people with concepts to do with school life led to a higher level of agreement with the minimalist view of Feng Shui. We discuss the implications of these findings for business practitioners.
\end{abstract}

Keywords: Feng Shui, instrumental view, spiritual view, minimalist view, priming

\section{Introduction}

Feng Shui is a system of beliefs stemming from ancient Chinese culture about how the environment and humankind interact (Koh, 2003; Kwok \& O'Brien, 1991; Rossbach \& Lin, 1998). Many Chinese practice Feng Shui and are willing to spend money on it and it is also gaining acceptance in the West (Tsang, 2004). A highly contentious example was the HK\$181,000 (approximately US\$23,200) spent by the 
Hong Kong Applied Science and Technology Research Institute (ASTRI) on Feng Shui (The Standard, 2007). ASTRI is a government-funded research institute. The media criticized public money being used on Feng Shui. At the heart of the debate was the general opinion that Feng Shui is superstitious and should not be practiced by ASTRI scientists, who are supposed to be rational. The head of ASTRI, Dr. Robert Yang, resigned in the midst of public pressure.

Different views of Feng Shui affect how likely people are to practice it. Yet, the scientific inquiry into views about Feng Shui is only just beginning. Luk et al. (2010) proposed a tripartite model of views about Feng Shui. They developed a psychometric scale for measuring these views and identified some of the dispositional determinants, including "internal locus of control," "external locus of control in chance," "external locus of control in powerful others," and "connectivity with nature." But for those wanting to change the environmental behavior of others, situational determinants are more interesting because these determinants can be manipulated. The present experiment is the first attempt to examine this possibility. We investigated the effect of one type of situational factor, conceptual primes. In the remainder of this paper, we review the relevant literature and develop our hypotheses. We then report the details of our experiment and discuss the implications of our findings.

\section{Views of Feng Shui}

In Chinese, Feng means wind and Shui means water. Literally, Feng Shui refers to the landscape of a location. There have been several Feng Shui theories (Koh, 2003; Rossbach \& Lin, 1998). Common to the different theories of Feng Shui is the notion that tangible components of the landscape, such as mountains or rivers, can generate an invisible energy, known as chi. Chi can be auspicious or inauspicious, depending on the position of the tangible components and their orientation (Koh, 2003; Rossbach \& Lin, 1998). To achieve good Feng Shui, the tangible components and the chi of a landscape should be balanced and in harmony. Good Feng Shui also reflects harmony between the environment and the inhabitants. This harmony would be internalized as harmony of the mind, which according to Chinese medicine theory is a source of health and longevity.

Although Feng Shui has a long history, it has rarely been tested scientifically. It is based upon mystical principles of how the universe works, and as a result is open to diverse interpretations even among Chinese. While some may believe that Feng Shui is useful, others see it as an art. Building on the works of Stokol (1990) and Tsang (2004), Luk et al. (2010) proposed that there are three views commonly held about Feng Shui: the instrumental view, the spiritual view, and the minimalist view. The instrumental view of Feng Shui is the belief that practicing Feng Shui can improve luck, health, wealth and interpersonal relationships. People who hold the instrumental view are more likely to make causal attributions to external factors (Luk et al., 2010), reflecting a perceived lack of personal control over events. This view has a strong positive effect on the intention to practice Feng Shui and make use of Feng Shui to improve luck, health, wealth and interpersonal relationships (Luk et al., 2010). 
The spiritual view considers Feng Shui to be an art form for living in harmony with the environment. According to this view, a harmonious environment is not a means but an end in itself. This view focuses on how Feng Shui arrangements inspire spiritual life and strengthen cultural identification. By practicing Feng Shui, people can develop a sense of oneness with the environment, derive spiritual satisfaction and attain a deep appreciation of Chinese culture. Supposedly, if the environment and its inhabitants are in harmony, inhabitants will feel good and their psychological wellbeing will be an added bonus. The spiritual view is positively correlated with Dutcher, Finley, Luloff, and Johnson's (2007) construct of connectivity with nature. It, too, has a positive impact on the intention to practice Feng Shui, but the impact is less strong than that of the instrumental view (Luk et al., 2010).

Finally, the minimalist view regards Feng Shui as superstitious and useless, and something to be ignored. Those who criticized the former chief executive of ASTRI for his spending on Feng Shui might espouse this view (e.g., Vines, 2007). Luk et al. (2010) have found that the adoption of this view by Chinese people is positively correlated with the adoption of a Western religion (e.g., Christian denominations, including Catholicism) and negatively correlated with the adoption of an Oriental religion (e.g., Taoism or Buddhism). Therefore, this view is likely to have been brought to the Chinese from the West, and is thus relatively new compared to the instrumental and spiritual views.

\section{Priming as a Situational Determinant}

The instrumental and the spiritual views of Feng Shui were rooted in Chinese culture and philosophies. Most Chinese are familiar with these two views. The minimalist view is likely to be more familiar to people who have received a Western education that emphasizes rational and scientific thinking. Therefore, for any Chinese receiving a Western education, or for Westerners learning Chinese culture, all three views will be familiar to them. Yet, all three views will not necessarily be salient for these individuals at the same time. The view which is the most accessible in memory is the one most likely to be salient to them and the one they are most likely to agree with. According to theory of social cognition, concept accessibility can be temporarily raised by the technique of "priming" (e.g., Anderson, Moskowitz, Blair, \& Nosek, 2007; Wyer, 2008). This theory indicates that we can prime some concepts related to a particular view of Feng Shui, thereby increasing the accessibility of this view of Feng Shui for a particular individual. As a result, the activated view would be the one most supported, and behavioral intention to practice Feng Shui would also change.

Priming refers to the unobtrusive activation of a concept already stored in memory. A concept that is primed would serve as a frame for subsequent information processing, and influence the interpretation of incoming information in a way consistent with the primed concept (Forster \& Liberman, 2007). There is a growing body of empirical research on how priming affects attitudes and preferences (e.g., Shen \& Wyer, 2008; Wheeler \& Berger, 2007). For example, in a recent experiment conducted by Wheeler and Berger (2007), the concept of shopping was primed by having participants write a story about shopping. In a second, ostensibly unrelated choice task male participants made more purpose-driven choices whereas female participants made more 
possibility-driven choices. The reason for this finding is that the concept of shopping is associated with problem-solving for men, but with exploration for women.

To activate a particular view of Feng Shui, we need to prime the concepts that are associated with that view. As already stated, people who hold the instrumental view tend to lack a sense of personal control over events. Thus, introducing concepts related to uncertainties and the ups and downs in life may induce this perceived lack of personal control over events and activate the instrumental view of Feng Shui.

On the other hand, people who hold a spiritual view of Feng Shui tend to have an appreciation of, and respect for, nature and for their own cultural identity. References to nature and to a sense of cultural identity would remind people of these concepts. After they are thus reminded, the spiritual view would come to the fore (i.e., become more accessible from memory) and become their guiding principle when making a judgment about Feng Shui.

Finally, the participants of the present experiment were university students educated in Hong Kong. They had been receiving Western education, which emphasizes rational thinking and denounces superstitions. If concepts associated with their school life are primed among them, the essence of the education they had received would be recalled. They would then turn to that mindset, and try to be more rational and less superstitious. As a consequence, they would agree more with the minimalist view of Feng Shui.

Our hypotheses are summarized as follows:

Prediction 1: After the concepts of uncertainties and ups and downs in life are primed, participants will have a higher level of agreement with the instrumental view of Feng Shui than with the other two views of Feng Shui.

Prediction 2: After the concepts of nature and cultural identity are primed, participants will have a higher level of agreement with the spiritual view of Feng Shui than with the other two views of Feng Shui.

Prediction 3: After concepts about school life are primed, participants (who are university students receiving Western education) will have a higher level of agreement with the minimalist view of Feng Shui than with the other two views of Feng Shui.

Past research has found that intention to practice Feng Shui is the strongest when consumers adopt the instrumental view, and is only moderately strong when they adopt the spiritual view (Luk et al., 2010). Luk et al. (2010) have also found that the intention to practice Feng Shui is negatively related to the minimalist view. If Predictions 1, 2, and 3 are valid, we can expect that an intention to practice Feng Shui would also vary after different concepts related to Feng Shui are primed. This gives rise to our last prediction: 
Prediction 4: Intention to practice Feng Shui is strongest after the concepts of uncertainties and ups and downs in life are primed, is moderately strong after the concepts of nature and cultural identity are primed, and is weakest after the concepts of school life are primed.

\section{Method}

\section{Participants}

Participants were 161 full-time university students from the business school of a major university in Hong Kong. Sixty three were male and 98 were female. Ages ranged from 17 to 29, with an average age of 21.89 and a standard deviation of 1.96. All were ethnic Chinese. They were knowledgeable about Chinese culture (which was included in their curriculum) and were able to comprehend the questionnaire items about Feng Shui. They participated in the research in exchange for extra course credit. Yet, participation was anonymous.

\section{Experimental Design}

The experiment had a between-participants design with three experimental conditions: (a) priming the concepts of uncertainties and ups and downs in life, (b) priming the concepts of nature and cultural identity, and (c) priming the concepts about school life. There are many priming methods in psychological literature. We chose Strauman and Higgins's (1987) priming method of sentence-completion task, in which participants are instructed to complete sentences that begin with certain concepts. The concepts that begin the incomplete sentences are automatically activated. This method is unobtrusive, and can be used to activate abstract concepts that are not linked to any visible icon or image. The sentence-completion task is similar to Wheeler and Berger's (2007) priming technique of story writing. Each participant of our experiment had to complete four sentences, each of which began with a specially chosen phrase. The phrases that began the sentences varied depending on the different experimental conditions.

In the experimental condition in which concepts of uncertainties and ups and downs in life were primed, the four phrases that began the incomplete sentences were:

1) "fortune and misfortune"

2) "fate and destiny"

3) "good luck and bad luck"

4) "ups and downs in life."

In the experimental condition in which concepts of nature and cultural identity were primed, the phrases that began the incomplete sentences were:

1) "harmonious environment"

2) "traditional culture"

3) "nature and man"

4) "spiritual life." 
For participants who were assigned to the school life concepts condition, the four phrases that began the incomplete sentences were:

1) "school life"

2) "the internet"

3) "sports"

4) "the current life stage."

These 12 concepts were identified in our pre-test, using student respondents. The dependent variables were the three views of Feng Shui and the intention to practice Feng Shui. All four variables were measured with the scales developed by Luk et al. (2010). There were 20 items altogether. The respondents made their responses to these items on a 6-point Likert scale, where 1 stood for "strongly disagree" and 6 stood for "strongly agree."

\section{Procedure}

Participants were recruited in class. They were given a booklet containing several questionnaires from ostensibly unrelated studies. The sentence completion tasks were one of them. Participants were assigned to one of the three experimental conditions by block randomization. At the end of the booklet, the scales measuring the three views of Feng Shui and intention to practice Feng Shui were attached. There were six possible orders in which the scales measuring the three views of Feng Shui could be presented. To counter-balance any order effect, one of the six orders was used to arrange these three scales in the booklet given to each participant.

The booklet also asked the participants to write down some personal information including age, sex, major subject, and religion. After all participants had returned the completed questionnaires, they were debriefed. No one questioned the true purpose of the experiment as they were filling out the questionnaires.

A total of 194 questionnaires were returned. Twenty one participants did not complete all four sentence completion tasks and were excluded from our analyses. Furthermore, religious beliefs have been found to have a strong effect on attitudes toward Feng Shui (Luk et al., 2010). We wanted to control for the effect of this extraneous variable by including only those who were not religious. Twelve participants indicated in the booklet that they were religious, and their data was excluded from sequent analyses. These two selection criteria reduced the number of usable questionnaires to 161 . 


\section{Results}

Table 1: Exploratory Factor Analysis on the Dependent Variables $(N=161)$

Statement 1 2 3

\section{Intention to Practice Feng Shui}

I will consult Feng Shui masters for advice. $\quad .81$

I will install a Feng Shui setting in my .77

residence.

I will install a Feng Shui setting in my .75

workplace.

I will buy and carry some Feng Shui objects

on my person.

I will buy Feng Shui guide books and decorate my residence and workplace according to their suggestions.

\section{Minimalist View of Feng Shui}

The choice and decoration of a residence/workplace should be based on aesthetic principles. Feng Shui can be ignored.

The choice and decoration of the residence/workplace should be based on pragmatic principles. Feng Shui can be ignored.

The choice and decoration of the residence/workplace should be based on principles of environmental protection. Feng Shui can be ignored.

Feng Shui is purely a superstition.

Feng Shui effects are purely psychological.

\section{Spiritual View of Feng Shui}

Feng Shui enhances my understanding of the human-environment relationship.

Feng Shui enhances my understanding of

Chinese culture.

Making good use of Feng Shui brings me

closer to nature.

Feng Shui enriches my spiritual life.

Feng Shui is the art of living in harmony with the environment. 
Table 1 (continued)

\section{Instrumental View of Feng Shui}

Living or working in locations with good

Feng Shui will bring good luck.

Making good use of Feng Shui can make the

residence/workplace more comfortable.

One can have good luck by choosing and

decorating the residence/workplace

according to Feng Shui principles.

Feng Shui can change one's destiny.

Making good use of Feng Shui can help me

do well.

\begin{tabular}{lcccc} 
\%o of variance of unrotated solution & 30.60 & 13.56 & 9.43 & 7.71 \\
Eigenvalue & 6.12 & 2.71 & 1.89 & 1.54 \\
& .85 & .83 & .80 & .80 \\
Mean & 2.96 & 3.72 & 3.62 & 3.68 \\
Standard deviation & .93 & .79 & .80 & .66 \\
\hline
\end{tabular}

Note: Factor loadings below .50 are suppressed.

Before testing our predictions, we ran an exploratory factor analysis on the four dependent variables to examine their dimensionality. Four factors emerged with eigenvalues over 1 . The scree plot supported a four-factor solution. All items fell into the factors to which they were supposed to belong. These factors explained $61.31 \%$ of the total variance, whereas the first factor explained $30.60 \%$ of the variance in the unrotated solution. Table 1 displays all the items of the four factors.

Table 2: Degree of Agreement With the Three Views of Feng Shui in Each Experimental Condition

Type of Primed Concepts

\begin{tabular}{lcccccc}
\cline { 2 - 6 } & $\begin{array}{c}\text { Uncertainties and } \\
\text { Ups and Downs in } \\
\text { Life } \\
(\boldsymbol{N = 5 6 )}\end{array}$ & $\begin{array}{c}\text { Nature and } \\
\text { Cultural Identity } \\
(\boldsymbol{N}=\mathbf{5 3})\end{array}$ & $\begin{array}{c}\text { School Life } \\
(\boldsymbol{N}=\mathbf{5 2})\end{array}$ \\
\hline $\begin{array}{l}\text { View of } \\
\text { Feng Shui }\end{array}$ & Mean & SD & Mean & SD & Mean & SD \\
\hline $\begin{array}{l}\text { Instrumental } \\
\text { View }\end{array}$ & 3.88 & .72 & 3.65 & .55 & 3.50 & .64 \\
$\begin{array}{l}\text { Spiritual } \\
\text { View }\end{array}$ & 3.62 & .86 & 3.82 & .54 & 3.43 & .90 \\
$\begin{array}{l}\text { Minimalist } \\
\text { View }\end{array}$ & 3.61 & .75 & 3.75 & .56 & 3.80 & 1.01 \\
\hline
\end{tabular}

Table 2 shows the degree of agreement with the three views of Feng Shui in each of the three experimental conditions. We ran a 3 (types of primes, a between-participants 
factor) by 3 (views of Feng Shui, a within-participants factor) repeated-measures ANOVA to test our predictions. The main between-participant effect of type of primes was not significant, $F(2,158)=1.20, p=.14$, partial eta squared $=.03$, meaning that none of the three types of primed concepts had a stronger or weaker effect than any other, and this was the case for all three attitudes toward Feng Shui. Also, the main within-participant effect of attitudes toward Feng Shui was not significant, $F(2,316)=.79, p=.46$, partial eta squared $=.01$, meaning that none of the three attitudes toward Feng Shui were any more or less accepted than any other for all participants.

More importantly, the interaction of type of primes applied on participants and their subsequent view of Feng Shui was significant, $F(4,316)=3.11, p=.02$, partial eta squared $=.04$. This significant interaction means that the pattern of agreement with the three views of Feng Shui differed across the three types of primed concepts. As shown in Figure 1, the patterns of the means across the three experimental conditions were in line with our first three predictions. To examine whether these differences were statistically significant, we followed Howell's (1997) suggestion (see also Boik, 1981) and ran three sets of simple effect tests. Each set of simple effect tests included two within-participant planned comparisons in each experimental condition, as described below. Using a familywise $\alpha$ of .05 for each set of simple effect tests, each planned comparison should have an $\alpha$ of .025.

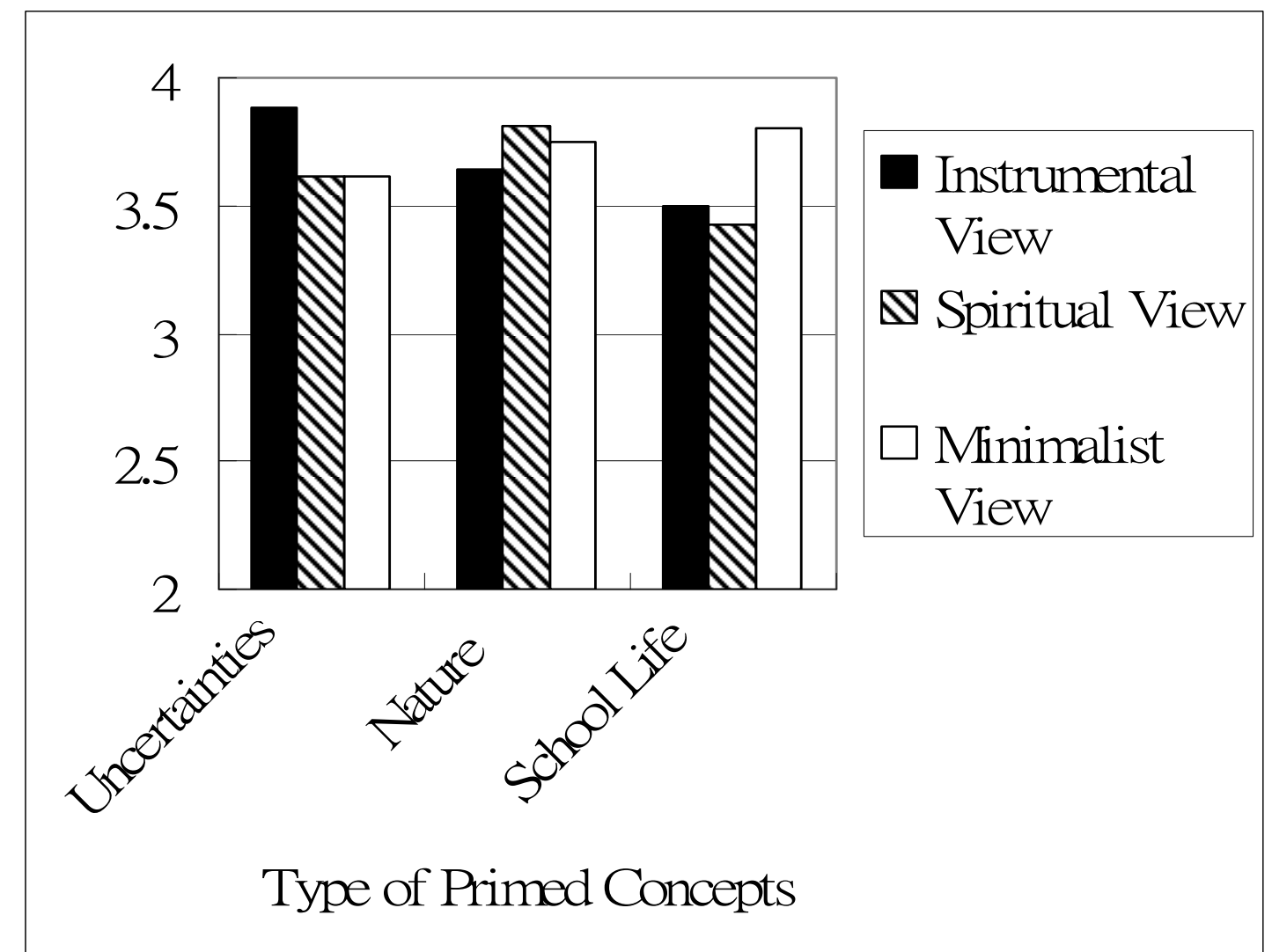

Figure 1: Degree of agreement with the three views of Feng Shui in each experimental condition. 
Note: "Uncertainties" = experimental condition in which concepts of uncertainties and ups and downs in life were primed; "Nature" = experimental condition in which concepts of nature and cultural identity were primed; "School Life" = experimental condition in which concepts of school life were primed.

For those primed with the concepts of uncertainties and of the ups and downs in life, we conducted two paired $t$-tests. The first paired $t$-test compared the level of agreement with the instrumental view (mean $=3.88, S D=.72$ ) with the level of agreement with the spiritual view (mean $=3.62, S D=.86$ ). The second compared the level of agreement with the instrumental view with the level of agreement with the minimalist view (mean $=3.61, S D=.75$ ). Because our predictions are directional, one-tailed tests would be appropriate. We found that both paired $t$-test were significant. In the first paired $t$-test, $t=2.66, d f=55, p<.01$, one-tailed. The second paired $t$-test approached statistical significance, $t=1.86, d f=55, p<.05$, one-tailed. Therefore, Prediction 1 was quite clearly supported.

For those primed with the concepts of nature and cultural identity, we conducted a paired $t$-test to compare the level of agreement with the spiritual view (mean $=3.82$, $S D=.54$ ) with the level of agreement with the instrumental view (mean $=3.65, S D=$ .55). This $t$-test was significant $(t=2.37, d f=52, p<.01$, one-tailed). We then conducted another paired $t$-test to compare the level of agreement with the spiritual view with the level of agreement with the minimalist view (mean $=3.75, S D=.56$ ). This second paired $t$-test was not significant $(t=.59, d f=52, p>.10)$. Therefore, Prediction 2 was only partially supported.

For those primed with the concepts of school life, we conducted a paired $t$-test to compare the level of agreement with the minimalist view (mean $=3.80, S D=1.01$ ) with the level of agreement with the instrumental view (mean $=3.50, S D=.64)$. The difference was not significant $(t=1.53, d f=51, p>.05$, one-tailed). Another paired $t$ test was run to compare the level of agreement with the minimalist view with the level of agreement with the spiritual view (mean $=3.43, S D=.90)$. The difference approached statistical significance $(t=1.80, d f=51, p<.05$, one-tailed). Therefore, Prediction 3 was only partially supported.

Finally, we compared intention to practice Feng Shui across the three experimental conditions by one-way ANOVA and found a significant effect, $F(2,158)=3.14, p=$ .05 , partial eta squared $=.04$. But the pattern of the mean differences was not totally in line with Prediction 4. As expected, intention to practice Feng Shui was weakest after the concepts of school life were primed (mean $=2.70, S D=.13$ ), but was stronger after the concepts of nature and cultural identity were primed (mean $=3.12$, $S D=.13$ ) than after the concepts of uncertainties and ups and downs in life were primed (mean $=3.05, S D=.12$ ). Post hoc tests showed that intention to practice Feng Shui was significantly $(p<.05$, one-tailed) lower in the school life concepts prime condition than in the nature and cultural identity concepts prime condition. Intention to practice Feng Shui was not significantly different whether the concepts of nature and cultural identity or those of uncertainties and ups and downs in life were primed. Therefore, Prediction 4 was also partially supported. 


\section{Discussion}

Building upon the work of Luk et al. (2010), we argue that each of the three views of Feng Shui (i.e., the instrumental, the spiritual, and the minimalist views) can be activated by getting Chinese students to contemplate different life-approach concepts before being asked about their attitudes toward Feng Shui. Having people think about a concept is a method known as "priming." To test our predictions, we ran an experiment. As predicted, priming the concepts of uncertainties and of the ups and downs in life led to a higher level of agreement with the instrumental view of Feng Shui. Priming the concepts of nature and cultural identity led to a higher level of agreement with the spiritual view of Feng Shui. And priming the concepts of school life led to a higher level of agreement with the minimalist view of Feng Shui. But intention to practice Feng Shui proved not to be the strongest after priming the concepts of uncertainties and the ups and downs in life. Although not all of our predictions were supported, the overall pattern of our findings is promising.

The most robust finding of our experiment was that priming the concepts of uncertainties and the ups and downs in life led to an increase in the level of agreement with the instrumental view of Feng Shui significantly above the levels of agreement with the other two views of Feng Shui. The ease with which the instrumental view of Feng Shui can be activated is perhaps due to the anxiety aroused by introducing ideas around the uncertainties and ups and downs of life. Out of a basic instinct to weather the difficulties in life, people tend to search quickly for uncertainty-reducing solutions. For Chinese people, Feng Shui is one of the options that may come to mind right away. Thus, negative affects may facilitate the capacity of priming to influence attitudes. Indeed, this negativity bias has been welldocumented in psychological literature (e.g., Shen \& Wyer, 2008). Our findings may be attributed to this tendency.

Relatively speaking, priming the other two types of concepts (the concepts of nature and cultural identity and the concepts of school life) was less effective in changing the levels of agreement with differing views of Feng Shui. Following the line of our argument in the preceding paragraph, these two types of concept are less affect-laden and do not have the same facilitating effect as that of the concepts of uncertainties and the ups and downs in life. We suggest that future research be done to verify this argument.

But the intention to practice Feng Shui proved not to be strongest after the concepts of uncertainties and the ups and downs in life were primed. We suspect that intention to practice Feng Shui was a function of a number of factors in addition to the primed instrumental view of Feng Shui. University students might be inherently resistant to the idea of practicing Feng Shui for improving their luck because they might try to avoid appearing superstitious or weak.

\section{Practical Implications}

Feng Shui is a situation that can be man-made, natural, or both. However, Feng Shui has by far no place in the literature on human-situation interaction. We believe that Feng Shui will gradually make an appearance in mainstream research on interactional 
psychology because it is becoming a global phenomenon. Buildings are designed according to Feng Shui rules (e.g., Brown, 2006; Rew, 2000; Weltman \& Hayes, 2005), and products and services are packaged with Feng Shui concepts included (e.g., JF Asset Management, 2008). Although it is unclear whether Feng Shui can really improve luck, health, or performance, people who agree with the instrumental or the spiritual views of Feng Shui would certainly be happier living or working in places with good Feng Shui. Feng Shui may be one of the factors that affect subjective well-being and possibly performance.

Furthermore, attitudes toward Feng Shui can be classed as types of environmental attitudes and behaviors. This is especially true of the spiritual view of Feng Shui. Environmentally friendly designs and environmental protection may be more appealing to people who have this approach to Feng Shui. City planners especially in China should take this factor into consideration. To this end, the spiritual view of Feng Shui should be explored more often in the media. Over time, perhaps, the spiritual view might become a chronically accessible construct (e.g., Bargh, Lombardi, \& Higgins, 1988).

The present experiment has shown that attitudes toward Feng Shui are, to a certain extent, malleable and can be changed by temporarily presented situational cues. Although the effects may be temporary, the possibility of change opens up a new horizon for those who want to change others' environmental attitudes and behaviors. City planners, architects, marketers, and policy makers are among those who may want to change people's environmental attitudes and behaviors. Some knowledge about Feng Shui would probably be very helpful in their works. Yet, how the environment at large can benefit from our findings awaits further research.

\section{Limitations and Future Research Directions}

Not-withstanding its contributions and the present experiment has several limitations that should be addressed in future studies. Firstly, it assessed only the intention to practice Feng Shui. It did not verify whether participants' stated intention to practice Feng Shui was borne out by their subsequent behavior via, for example, renovations made in their home or work spaces with regard to Feng Shui principles. Future studies should address this by using a longitudinal research design.

Secondly, all participants of the present experiment were university students. The homogeneity of the sample was its strength in that it gave control over the confounding effects of extraneous variables such as age, socioeconomic status, and culture. But if we want to establish the external validity of the findings, the experiment should be replicated among non-university adolescents and adults. If the findings are consistent across different samples, we would be much more confident in stating that the conceptual primes are practical and are significant determinants of behaviors related to Feng Shui.

Finally, it would be useful to examine how the instrumental, spiritual, and minimalist views of Feng Shui are dependent on and can affect one's mental and/or physical health status. The research questions that deserve further exploration include, for example, whether people who adopt the instrumental view are less healthy -- mentally 
and/or physically -- than people who adopt one of the other two views. Would people who adopt the minimalist view ignore all alternative therapies when they have mental and/or physical health problems, and would people who adopt the instrumental or the spiritual views be more likely to try alternative therapies? Answers to these research questions would enhance the practical significance of this research, and would offer the potential to relate this research to other disciplines.

\section{Acknowledgement:}

This research was supported by a Strategic Research Grant (7001910-880) provided by the City University of Hong Kong for the third author.

\section{References}

Anderson, S. M., Moskowitz, G. B., Blair, I. V., \& Nosek, B. A. (2007). Automatic thought. In A. W. Kruglanski \& E. T. Higgins (Eds.), Social psychology: Handbook of basic principles $\left(2^{\text {nd }}\right.$ ed., pp. 138-175). New York: The Guilford Press.

Bargh, J. A., Lombardi, W. J., \& Higgins, E. T. (1988). Automaticity of chronically accessible constructs in person X situation effects on person perception: It's just a matter of time. Journal of Personality and Social Psychology, 55, 599-605.

Boik, R. J. (1981). A priori tests in repeated measures designs: Effects of nonsphericity. Psychometrika, 46, 241-255.

Brown, K. (2006). Using Feng Shui principles can create harmony among appliances in kitchen. Boulder County Business Report, 25, 7B.

Dutcher, D. D., Finley, J. C., Luloff, A. E., \& Johnson, J. B. (2007). Connectivity with nature as a measure of environmental values. Environment and Behavior, 39, 474-493.

Forster, J., \& Liberman, N. (2007). Knowledge activation. In A. W. Kruglanski \& E. T. Higgins (Eds.), Social psychology: Handbook of basic principles $\left(2^{\text {nd }}\right.$ ed., pp. 201231). New York: The Guilford Press.

Howell, D. C. (1997). Statistical methods for psychology (4 ${ }^{\text {th }}$ ed.). Belmont, CA: Duxbury Press.

JF Asset Management (2008). www.jfam.com. Retrieved April 22, 2008.

Koh, V. (2003). Basic science of Feng Shui: A handbook for practitioners. Singapore: ASIAPAC.

Kwok, M. H., \& O’Brien, J. (1991). The elements of Feng Shui. New York: Barnes \& Noble Books.

Luk, C. L., Wan, W. W. N., Chow, R. P. M., Chow, C. W. C., Fam, K. S., Wu, P., \& Kim, S. (2010). Consumers' views of Feng Shui: Antecedents and behavioral consequences. Forthcoming in Psychology \& Marketing. 
Rew, T. (2000). Feng Shui today: A guide to enriching your life. New York, NY: HBI.

Rossbach, S., \& Lin, Y. (1998). Feng Shui design: From history and landscape to modern gardens and interiors. London: Sidgwick \& Jackson.

Shen, H., \& Wyer, R. S. Jr. (2008). Procedural priming and consumer judgments: Effects on the impact of positively and negatively valenced information. Journal of Consumer Research, 34, 727-737.

Stokols, D. (1990). Instrumental and spiritual views of people-environment relations. American Psychologist, 45, 641-646.

Strauman, T. J., \& Higgins, E. T. (1987) Automatic activation of self-discrepancies and emotional syndromes: When cognitive structures influence affect. Journal of Personality and Social Psychology, 53, 1004-1014.

The Standard (2007). ASTRI boss quits in row over money for fung shui. Retrieved April 25, 2007, from

http://www.thestandard.com.hk/news_detail.asp?pp_cat=11\&art_id=42961\&sid=132 93654\&con type $=1$.

Tsang, E. W. K. (2004). Toward a scientific inquiry into superstitious business decisionmaking. Organization Studies, 25, 923-946.

Vines, S. (2007). Money to burn. South China Morning Post, April 27, 2007.

Weltman, B., \& Hayes, M. (2005). Feng Shui for beginners: An organized, harmonious work environment is a business asset. Journal of Accountancy, 200, 36-39.

Wheeler, S. C., \& Berger, J. (2007). When the same prime leads to different effects. Journal of Consumer Research, 34, 357-368.

Wyer, R. S. (2008). The role of knowledge accessibility in cognition and behavior: Implications for consumer information processing. In C. Haugtvedt, F. Kardes, \& P. Herr (Eds.), Handbook of consumer research (pp. 31-75). Mahwah, NJ: Erlbaum. 